\title{
Developing the tube theory for polymer knots
}

\author{
Liang Dai (1)* \\ Department of Physics, City University of Hong Kong, Hong Kong, China \\ and Shenzhen Research Institute, City University of Hong Kong, Shenzhen, China
}

(Received 7 January 2020; accepted 25 March 2020; published 16 April 2020)

\begin{abstract}
Entanglements make polymers fundamentally different from other molecules and thus are a major theme in polymer physics research. Interchain entanglements have been extensively investigated in past decades, while intrachain entanglements, often appearing as polymer knots, are much less understood. In this work, we apply the tube theory for polymer knots based on the tube model: the polymer segments in a knot core are confined within a tube due to topological entanglements. We use an approach of visualizing and quantifying the "tubes." First, we perform Monte Carlo simulations to generate a large number of polymer knots. Then, we superimpose knot cores to obtain average knot conformations. The fluctuations of individual knot conformations around average knot conformations produce tubes, which materialize the conceptual tubes. Analyzing the tubes validates many scaling relationships and determines the relevant parameters. Furthermore, we reveal a heart shape for polymer trefoil knots, which results from the competition of entropy and bending energy. Overall, this work builds the foundation of the tube theory for polymer knots.
\end{abstract}

DOI: 10.1103/PhysRevResearch.2.022014

Just like entanglements in everyday linear objects, entanglements are common in polymers. Entanglements are caused by the uncrossability of polymer segments and affect conformational and dynamic properties of polymers. Due to the dramatic effects of entanglements in polymer dynamics and rheology, interchain entanglements have received intensive investigation in past decades. One well-established theory to describe interchain entanglements is the tube theory, which assumes that a polymer is confined within a tube due to the entanglements with neighbor polymers [1]. Besides interchain entanglements, polymers can also form intrachain entanglements, which often appear as polymer knots [2-4] and are much less understood than interchain entanglements.

Recently, knots in DNA [5-10] and proteins [11-15] have been observed in many experiments. Many intriguing or even counterintuitive phenomena have been found for polymer knots [16-20]. The importance of knotting has been demonstrated by the effects of knotting on the rheology of knotted polymers [9,21-23], the biological functions of knotted DNA [24] and knotted proteins [25,26], and the catalysis of knotted molecules [27]. Quantitative description of these knotting effects requires an analytic theory for polymer knots, which is unavailable.

In this work, we apply the tube theory to polymer knots. The basic idea of the theory is the tube model, which assumes the polymer segments in a knot core are confined within a tube due to topological entanglements [16]. Applying this

\footnotetext{
*liangdai@ cityu.edu.hk

Published by the American Physical Society under the terms of the Creative Commons Attribution 4.0 International license. Further distribution of this work must maintain attribution to the author(s) and the published article's title, journal citation, and DOI.
}

tube model to derive properties of polymer knots relies on the validation of several assumptions and determination of many parameters, which are to be addressed using an effective approach in this work: materializing the "tubes" for polymer knots by computer simulations. The calculation of tubes based on primitive path analysis has been previously applied for interchain entanglements [28].

First, we obtain the tubes for polymer knots by simulations. We perform Monte Carlo simulations to generate linear polymer conformations under equilibrium and then analyze the knots inside the polymer conformations. We describe polymers by the discretized wormlike chain model, i.e., a string of beads with bending energies [17,29]. The bond length between two adjacent beads is fixed as $a$. A bending potential is applied for three adjacent beads to reproduce a persistence length, $L_{p}$ :

$$
E_{\text {bend }} /\left(k_{B} T\right)=(1 / 2)\left(L_{p} / a\right) \theta^{2},
$$

where $\theta$ is the bending angle, $k_{B}$ is the Boltzmann constant, and $T$ is the temperature. We do not apply any pairwise interaction between beads. The data shown in this Rapid Communication are based on the simulations with the persistence length $L_{p}=10 a$ and the polymer length $L=500 a=50 L_{p}$. We use $L_{p}$ as the unit length. As shown by additional results in the Supplemental Material [30], the simulation results of polymer knots are insensitive to $a / L_{p}$ and $L / L_{p}$ when $a / L_{p} \leqslant 1 / 4$ and $L / L_{p} \geqslant 50$. Note that this work focuses on the investigation of the knots in long wormlike chains. Our parameters of $a / L_{p}=1 / 10$ and $L / L_{p}=50$ can substantially eliminate the effects caused by discretization and finite chain lengths.

We calculate average knot conformations using the following steps. First, we generate single polymer conformations by performing PERM (pruned-enriched Rosenbluth method) 
(a)

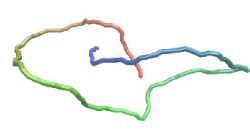

(b)

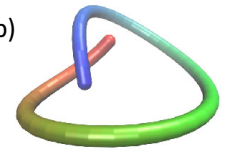

(d)
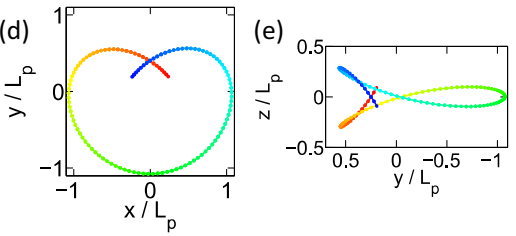

(f)

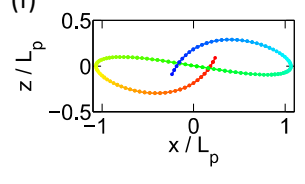

(c)

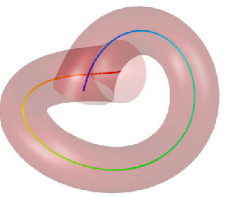

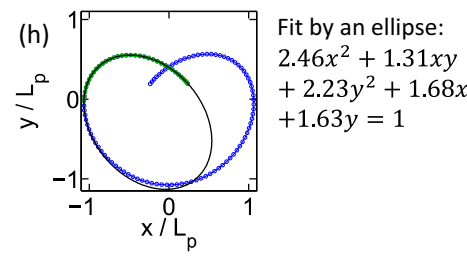

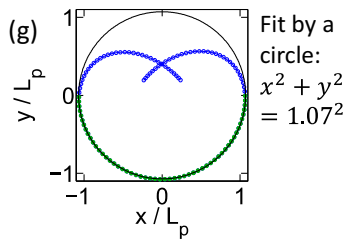

FIG. 1. (a) Conformations of the knot cores in left-handed trefoil-knotted polymers generated by Monte Carlo simulations. The contour lengths of the knot cores are $L_{\mathrm{knot}}=12 L_{p}$, where $L_{p}$ is the persistence length. (b) The average knot conformation obtained by superimposing $10^{5}$ conformations. (c) The tube has the same shape as the left cartoon, while the diameters of the tube correspond to the standard deviations of the fluctuations of individual knot-core conformations around the average knot conformation. Note that the diameter varies along the tube axis. (d)-(f) Projections of the average knot conformation along three directions. (g) The bottom part of the $x-y$ projection is fit by a circle. (h) The top-left part of the $x-y$ projection is fit by an ellipse.

simulations. PERM is a sequential Monte Carlo algorithm that has been demonstrated to be highly efficient in generating polymer conformations [31-33]. Second, we identify the knot type for each polymer conformation through the calculation of the Alexander polynomials $[17,34,35]$. We employ the minimally interfering closure scheme to connect polymer ends before the calculation of the Alexander polynomials [36]. We determine the knot core by cutting beads from both polymer ends until the change in the knot type. The contour length of the knot core is defined as the knot size, $L_{\text {knot }}$. Figure 1(a) displays three examples of knot-core conformations. Third, we superimpose the knot-core conformations with the same knot size $L_{\text {knot }}$ to obtain the average conformation for a given $L_{\mathrm{knot}}$. Before superimposition, we fit the conformations through rotation and translation to minimize the RMSD (rootmean-square deviation). Note that because trefoil knots can be left-handed or right-handed, averaging trefoil-knot conformations with both handedness would remove the structural features of trefoil knots. As a result, we make the average only for the knot-core conformations with the same handedness.

Figure 1(b) shows the average conformation for a trefoilknot core with the knot size $L_{\text {knot }}=12 L_{p}$, which is the most probable knot size $[17,37]$. The coordinates of this average knot conformation and the fits by empirical equations are included in the Supplemental Material [30]. Figures 1(d)-1(f) show three projections. It is intriguing that the bottom part of the $x-y$ projection can be very well fit by a circle with a radius of $R_{\text {circle }} \approx 1.07 L_{p}$ [Fig. $1(\mathrm{~g})$ ]. In addition, the top-left part of the $x-y$ projection can be fit by an ellipse [Fig. 1(h)]. The average knot conformation exhibits a beautiful heart shape, which reveals important information about the competition of interactions in polymer knots $[16,38]$ and will be discussed later.

Figure 1(c) shows the tube for polymer knots. The axis of this tube follows the average knot conformation, while the diameters of the tube vary along the axis and correspond to the amplitudes of fluctuations of individual knot-core conformations around the average knot conformation.

Next, we analyze the tubes of polymer knots to facilitate the development of the tube theory for polymer knots. Applying the tube model to derive properties of polymer knots relies on several assumptions. Grosberg and Rabin [16] proposed that the free energy cost of knot formation $F_{\text {knot }}$ consists of two parts:

$$
F_{\text {knot }} \approx E_{\text {bend }}+F_{\text {entropy }},
$$

where $E_{\text {bend }}$ is the bending energy and $F_{\text {entropy }}$ is the free energy increase due to the entropic loss. The entropic loss is caused by the fact that polymer segments inside the knot core are restricted by the entanglements within the knot. Deriving the analytic expressions of $E_{\text {bend }}$ and $F_{\text {entropy }}$ requires several assumptions. In the following, we describe these assumptions and examine the validity of these assumptions by simulation results.

(i) The tube model assumes a uniform tube diameter $D_{\text {tube }}$, which is proportional to the knot size $L_{\text {knot }}$. To validate this assumption, we need to calculate $D_{\text {tube }}$. We quantify $D_{\text {tube }}$ by the amplitude of the fluctuations around the average knot conformation, i.e., RMSD. Figures 2(a) and 2(b) show the average knot conformations and RMSD for different knot sizes. RMSD varies along the tube axis. Because the variance is not significant, we quantify the tube diameter by the average RMSD:

$$
D_{\text {tube }} \equiv 2\langle\text { RMSD }\rangle \text {. }
$$

The prefactor 2 is added to take into account the fact that RMSD corresponds to the tube radius. The top inset of Fig. 2(c) shows that $D_{\text {tube }}$ is roughly proportional to the knot size $L_{\mathrm{knot}}$ :

$$
L_{\mathrm{knot}} \approx 20\langle\mathrm{RMSD}\rangle=10 D_{\text {tube }},
$$

and then we obtain the aspect ratio of the tube:

$$
p \equiv L_{\text {knot }} / D_{\text {tube }} \approx 10 .
$$

The aspect ratio is an important parameter to quantify the shape of polymer trefoil knots and derive many properties of polymer knots. In previous studies, the value of $p$ is estimated to be $p \approx 12.4$ based on the maximally inflated trefoil knot [39], or $p \approx 16$ based on the fit to the dependence of the knot size on the chain width [17].

(ii) To derive $F_{\text {entropy }}$, the tube model makes use of the Odijk scalings, which were originally derived for the extension and entropic loss of a polymer confined in a tube [40]. Note that the "tubes" for polymer knots are not real 
(a)
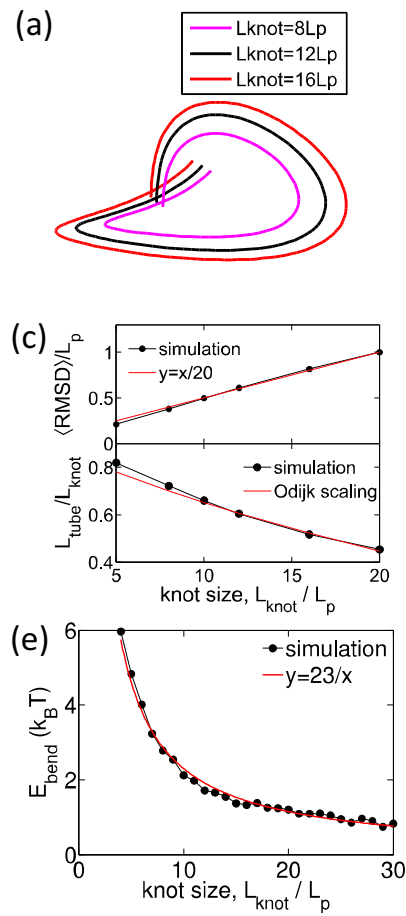

(b) $=$

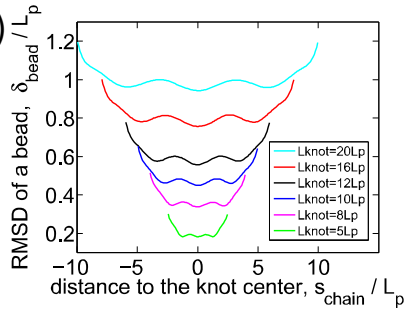

(d)

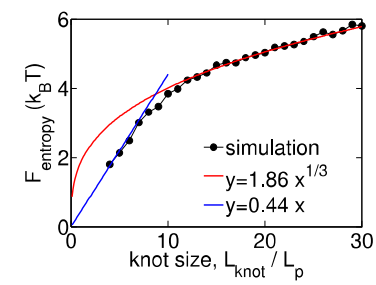

(f)

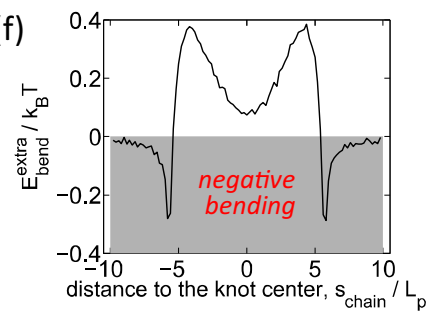

FIG. 2. (a) Average trefoil-knot conformations with different knot sizes. (b) Fluctuations of individual polymer knots around average knot conformations for different knot sizes. (c) Normalized RMSD and normalized length of the tube axis as a function of the knot size. The two fit lines are based on Eqs. (4) and (6). (d) The free energy increase due to entropic loss of the polymer segments in the knot core. (e) Extra bending energy induced by knotting as a function of the knot size. (f) Extra bending-energy density along a knot core for $L_{\mathrm{knot}}=12 L_{p}$. $E_{\mathrm{bend}}^{\text {extra }}$ is normalized to be the extra bending energy per unit length of $L_{p}$. The shaded area corresponds to "negative extra bending."

tubes with solid walls, and hence it is not clear whether the Odijk scalings are applicable in the tube model for polymer knots. To examine the applicability, we analyze $L_{\text {tube }} / L_{\text {knot }}$ and $F_{\text {entropy }}$. Here, $L_{\text {tube }}$ is the arc length of the tube axis, corresponding to the polymer extension in a tube. The bottom inset of Fig. 2(c) shows that $L_{\text {tube }} / L_{\text {knot }}$ roughly follows the Odijk scaling:

$$
L_{\text {tube }} / L_{\text {knot }} \approx 1-0.17 k_{1}\left(D_{\text {tube }} / L_{p}\right)^{2 / 3} \text { with } k_{1} \approx 2.05,
$$

where the coefficient 0.17 is the original coefficient in the Odijk scaling [41], and $k_{1}$ is an additional coefficient to take into account that the tube walls for polymer knots are softer than solid walls. Figure 2(d) shows the free energy increase due to the entropic loss in knots, which is calculated from simulation results through $F_{\text {entropy }}\left(L_{\text {knot }}\right) /\left(k_{B} T\right)=$ $-\ln \left[p_{\text {knot }}\left(L_{\text {knot }}\right)\right]-E_{\text {bend }}\left(L_{\text {knot }}\right) /\left(k_{B} T\right)$. Here, $p_{\text {knot }}$ is the probability distribution of the knot size in our simulations. We find that for $L_{\mathrm{knot}}>10 L_{p}, F_{\text {entropy }}$ roughly follows the Odijk scaling:

$$
\begin{aligned}
F_{\text {entropy }} /\left(k_{B} T\right) & \approx 1.86\left(L_{\text {knot }} / L_{p}\right)^{1 / 3} \\
& \approx 2.36 k_{2} L_{\text {knot }} D_{\text {tube }}^{-2 / 3} L_{p}^{-1 / 3} \text { with } k_{2} \approx 0.17,
\end{aligned}
$$

where 1.86 is obtained by fitting, 2.36 is the original coefficient in the Odijk scaling [41], and $k_{2} \approx 0.17$ is an additional coefficient to take into account that the tube walls are soft. In Eq. (7), we make use of $L_{\text {knot }}=p D_{\text {tube }}$ with $p=10$. For $L_{\text {knot }}<10 L_{p}, F_{\text {entropy }}$ significantly deviates from the Odijk scaling. Recall that the most probable trefoil knot size is $L_{\mathrm{knot}} \approx 12 L_{p}$, and around this knot size, Eq. (7) is applicable.

(iii) The tube model assumes the bending energy scales as $E_{\text {bend }} \sim L_{\mathrm{knot}} R_{\mathrm{knot}}^{-2} \sim L_{\mathrm{knot}}^{-1}$ based on the approximation that the radius of curvature $R_{\text {knot }}$ is proportional to $L_{\text {knot }}$. Figure 2(e) shows $E_{\text {bend }}$ follows

$$
E_{\text {bend }} /\left(k_{B} T\right) \approx 23\left(L_{\mathrm{knot}} / L_{p}\right)^{-1} .
$$

In addition to the total bending energy, we also analyze the bending energy distribution along the contour of the knot core as shown in Fig. 2(f). Here, we plot the extra bendingenergy density, which is defined as the difference in the bending-energy density between the knotted and unknotted segments:

$$
E_{\text {bend }}^{\text {extra }} \equiv E_{\text {bend }}^{\text {knot }}-\left\langle E_{\text {bend }}^{\text {unknot }}\right\rangle .
$$

There are two important features in Fig. 2(f). First, the bending-energy density varies greatly with the knot core. Second, some regions of the knot core possess negative extra bending energies. Note that the unknotted segments also possess certain bending energies due to thermal fluctuations. The negative $E_{\text {bend }}^{\text {extra }}$ indicates that the polymer segments at the boundaries of a knot core adopt less bent conformations than unknotted conformations. In other words, a knot suppresses the bending at boundaries. This observation is surprising but understandable. Straight conformations at the boundaries of a knot core should favor a larger separation between two segments at the entanglement site and then reduce the entropic loss, which will be discussed later. Nonuniform distributions of bending-in particular, the negative extra bending energies - should be the reason for knot localization on a polymer with nonuniform bending stiffness, which has been observed in previous studies $[42,43]$.

In addition to critically examining assumptions in the tube model, our simulations also reveal a heart shape of the average knot conformation. To clearly view the average knot conformation, we printed a 3D object for the knotted curve [Figs. 3(a)-3(c). In the following part, we will explain the shape of the average knot conformation based on the idea that this shape should minimize the free energy. We attempt to search the knotted curve with the minimum free energy and compare it with the average knot conformation.

Inspired by the half-circle shape in Fig. 1(g), we search the knotted curve with the minimum free energy using the following approach. First, we tie a knotted curve with the minimum bending energy, as shown in Fig. 3(d). To tie a knot, the total bending angle is at least $2 \pi$. One way to achieve the minimum bending angle is to make a knotted curve consisting of a nearly full circle and a twist part [Fig. 3(d)]. The twist part is necessary for knot formation. The total bending angle of the twist part can be minimized towards zero by reducing its size towards zero. For a given knot size of $L_{\mathrm{knot}}$, this knotted curve has the minimum bending energy $E_{\text {bend }}^{\text {min }} /\left(k_{B} T\right) \approx$ $2 \pi^{2} L_{p} / L_{\text {knot }}$. Note that the prefactor 23 in Eq. (8) is slightly larger than $2 \pi^{2} \approx 19.74$. 

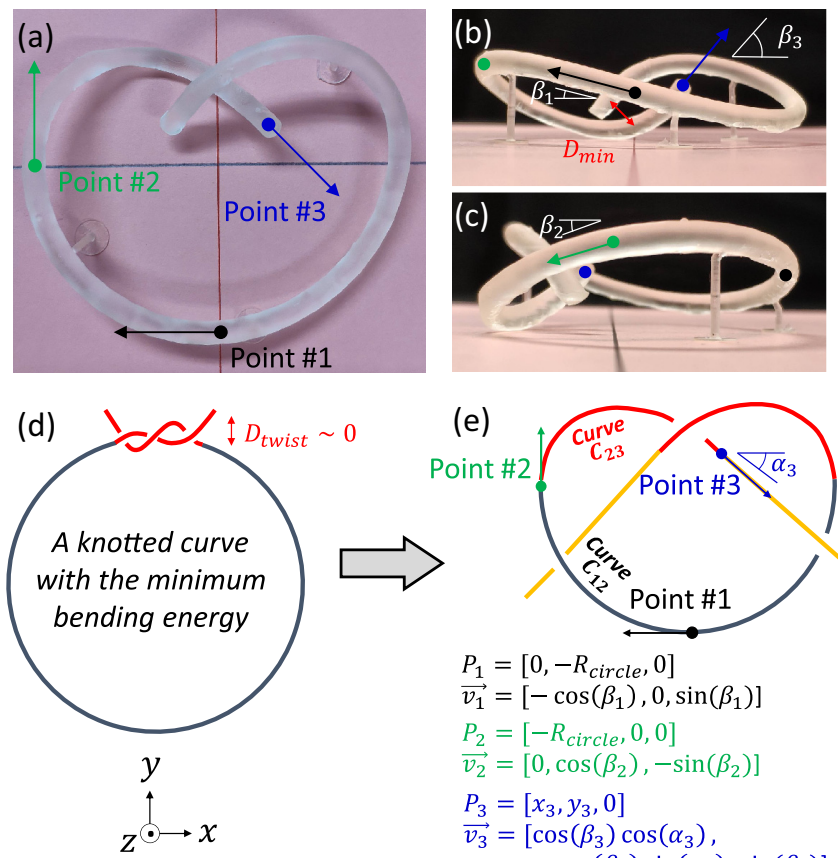

(e)

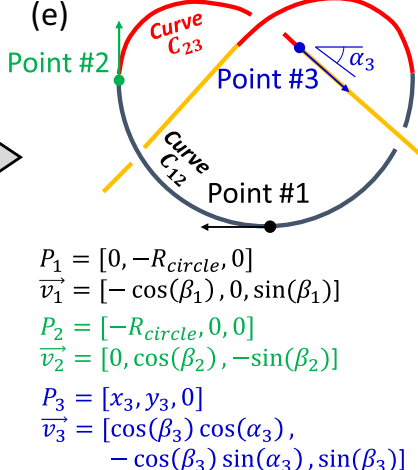

FIG. 3. (a)-(c) The top view and side views of a $3 \mathrm{D}$ printed object based on the average knot conformation with $L_{\mathrm{knot}}=12 L_{p}$. The minimum distance between the two end segments of the curve is denoted by $D_{\min }$. (d) A knotted curve with the minimum bending energy. (e) We distort the curve in (d) for the free energy minimization. Three points are defined on the curve to facilitate the formalization of the knotted curve. Based on these three points, we define two curves: $C_{12}$ and $C_{23}$. The two yellow lines are added to aid visualization of the knot.

Although the knotted curve in Fig. 3(d) can minimize the bending energy, this knotted curve is not favored because polymer segments in the twist part can only fluctuate within very small space, corresponding to a small entropy. Accordingly, our next step is to enlarge and distort the twisting portion of this knotted curve to increase the entropy for free energy minimization [Fig. 3(e)]. During this process, the halfcircle at the bottom of the curve is also slightly distorted to ensure the smooth bending and minimize the bending energy. To facilitate the optimization of the knotted curve, we generate the knotted curve based on the spline interpolation of three points. The first two points, $P_{1}=\left[0,-R_{\text {circle }}, 0\right]$ and $P_{2}=$ $\left[-R_{\text {circle }}, 0,0\right]$, are the lowest and the most left points of the circle, respectively. Here, $R_{\text {circle }}$ is the radius of the circle. For $L_{\text {knot }}=12 L_{p}$, we have $R_{\text {circle }} \approx 1.07 L_{p}$ [Fig. $\left.1(\mathrm{~g})\right]$. The third point, $P_{3}=\left[x_{3}, y_{3}, 0\right]$, is the end point of the curve, while the values of $x_{3}$ and $y_{3}$ are to be determined during the free energy minimization. Note that the average knot conformation has the rotational symmetry along the $y$ axis, and hence we only need to determine one half of the curve, i.e., from $P_{1}$ to $P_{2}$ (curve $C_{12}$ ), and from $P_{2}$ to $P_{3}$ (curve $C_{23}$ ).

Then, we proceed to the generation of the knotted curve based on the three points. To make smooth connections between $P_{1}$ and $P_{2}$ and between $P_{2}$ and $P_{3}$, we adopt the cubic Bezier curve, which is a widely used method to connect two points with given positions and orientations. We define the orientations at three points as shown in Fig. 3. Here, $\alpha_{3}$ represents the angle within the $x-y$ plane [Fig. 3(e)], and $\beta_{1}$, $\beta_{2}$, and $\beta_{3}$ represent the tilt angles with respect to the $x$ - $y$ plane (Fig. 3). Applying cubic Bezier curves, the connection curve $C_{12}$ between $P_{1}$ and $P_{2}$ follows

$$
\begin{aligned}
C_{12}(t)= & (1-t)^{3} P_{1}+3(1-t)^{2} t\left(P_{1}+k_{12} \overrightarrow{v_{1}}\right)+3(1-t) t^{2} \\
& \times\left(P_{2}-k_{21} \overrightarrow{v_{2}}\right)+t^{3} P_{2} \text { with } 0 \leqslant t \leqslant 1
\end{aligned}
$$

and the connection curve $C_{23}$ between $P_{2}$ and $P_{3}$ follows

$$
\begin{aligned}
C_{23}(t)= & (1-t)^{3} P_{2}+3(1-t)^{2} t\left(P_{2}+k_{23} \overrightarrow{v_{2}}\right)+3(1-t) t^{2} \\
& \times\left(P_{3}-k_{32} \overrightarrow{v_{3}}\right)+t^{3} P_{3} \text { with } 0 \leqslant t \leqslant 1
\end{aligned}
$$

Here, the coefficients $k_{12}, k_{21}, k_{23}$, and $k_{32}$ are adjustable parameters in cubic Bezier curves.

After parametrizing the knotted curve, we proceed to free energy minimization. To calculate the bending energy, we adapt Eq. (1) for a continuous curve

$$
E_{\text {bend }}^{\text {curve }} /\left(k_{B} T\right)=(1 / 2) L_{p} \int R_{\mathrm{c}}^{-2}(s) d s .
$$

The integration is over the arc of the curve and $R_{\mathrm{c}}$ is the radius of curvature. To calculate the entropic loss by knotting, we use the following approximation. For the polymer segments in $C_{12}$, we ignore the effect of knotting on the entropy, because these segments are far away from the entanglement site of the knot. For the polymer segments in $C_{23}$, we assume these segments are confined in a tube with a diameter of $D_{\min }$, where $D_{\text {min }}$ is the minimal distance between the two arms of the knot [see the illustration of $D_{\text {min }}$ in Fig. 3(b)]. Then, the free energy contributed by the entropic loss can be approximated by the Odijk scaling as mentioned above [40]:

$$
F_{\text {entropy }} /\left(k_{B} T\right) \approx k_{\text {entropy }} L_{23} D_{\min }^{-2 / 3} L_{p}^{-1 / 3},
$$

where $k_{\text {entropy }}$ is a prefactor to be determined, and $L_{23}$ is the arc length of the curve $C_{23}$. Based on the above equation, the entropic loss mainly depends on $D_{\min }$, because the confinement effect comes from the entanglement site. Straightening the conformations of the segments around the entanglement site allows more translational freedom of these segments, which should be the reason for the negative extra bending energies observed in Fig. 2(f).

With the functional forms in Eqs. (12) and (13), we search the knotted curve with the minimum free energy by adjusting the parameters: $\beta_{1}, \beta_{2}, \beta_{3}, \alpha_{3}, x_{3}, y_{3}, k_{12}, k_{21}, k_{23}$, and $k_{32}$. In addition, we tune $k_{\text {entropy }}$ in Eq. (13) to match the knotted curve from the free energy minimization and the average knot conformation from simulations [Fig. 4(b)]. This fit yields $k_{\text {entropy }} \approx 0.45$. It is interesting to see the competition of different parts of the knotted curve during free energy minimization. Figure 4(a) shows the free energies as a function of $\beta_{2}$, the tilt angle at point 2 . Curve $C_{12}$ prefers a smaller $\beta_{2}$ to minimize the bending energy, while curve $C_{23}$ prefers a larger $\beta_{2}$ to minimize the free energy (mainly entropic contribution). The competition leads to a free energy minimum at $\beta_{2}=16^{\circ}$ in agreement with the average knot conformation.

In conclusion, we build the foundation of the tube theory for polymer knots by critically examining the assumptions and determining the parameters, including the aspect ratio of the tube $p \approx 10$ and the prefactors of $E_{\text {bend }}$ and $F_{\text {entropy }}$. 
(a)

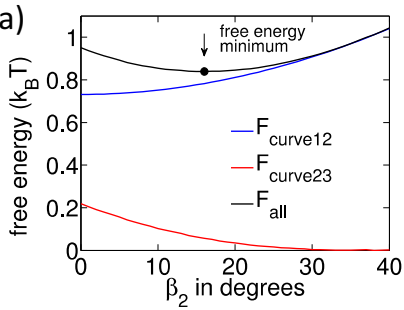

(b)

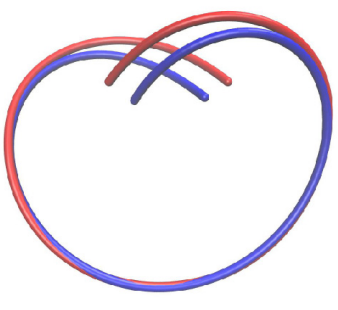

FIG. 4. (a) Free energies of the curves $C_{12}$ and $C_{23}$ and their sum as a function of the angle $\beta_{2}$. The free energy of the curve $C_{23}$ is offset to make the last data point at $y=0$. (b) Comparison of the average knot conformation from simulation (blue) and the knotted curve from the free energy minimization (red).

We also determine the applicable regime of the tube theory: $L_{\text {knot }}>10 L_{p}$. Meanwhile, we discover a surprising result: negative extra bending energies at knot boundaries, which can explain knot localization observed in many previous studies. Furthermore, we obtain and explain the heart shape of polymer trefoil knots, which reveals the delicate competition of the bending energy and entropy in a knot core. The results of this work can be applied to calculate many properties of polymer knots, including free energy of knots, knotting probability, knot sizes, and knot shape, and guide the rational control of polymer knots, such as knot localization, by tuning nonuniform bending stiffness. Looking forward, it should be interesting to calculate average knot conformations for other knot types, which requires more computational effort due to the lower probabilities of other knot types.

This research is financially supported by City University of Hong Kong (Projects No. 9610420 and No. 7005266), the National Natural Science Foundation of China (Project No. 21973080), and Guangdong Basic and Applied Basic Research Fund (Project No. 2019A1515011736).
[1] P.-G. de Gennes, J. Chem. Phys. 55, 572 (1971).

[2] C. Micheletti, D. Marenduzzo, and E. Orlandini, Phys. Rep. 504, 1 (2011).

[3] E. Orlandini, J. Phys. A 51, 053001 (2017).

[4] P. Virnau, Y. Kantor, and M. Kardar, J. Am. Chem. Soc. 127, 15102 (2005).

[5] V. V. Rybenkov, N. R. Cozzarelli, and A. V. Vologodskii, Proc. Natl. Acad. Sci. USA 90, 5307 (1993).

[6] X. R. Bao, H. J. Lee, and S. R. Quake, Phys. Rev. Lett. 91, 265506 (2003).

[7] E. Ercolini, F. Valle, J. Adamcik, G. Witz, R. Metzler, P. De Los Rios, J. Roca, and G. Dietler, Phys. Rev. Lett. 98, 058102 (2007).

[8] C. Plesa, D. Verschueren, S. Pud, J. van der Torre, J. W. Ruitenberg, M. J. Witteveen, M. P. Jonsson, A. Y. Grosberg, Y. Rabin, and C. Dekker, Nat. Nanotechnol. 11, 1093 (2016).

[9] S. Amin, A. Khorshid, L. Zeng, P. Zimny, and W. Reisner, Nat. Commun. 9, 1506 (2018).

[10] R. K. Sharma, I. Agrawal, L. Dai, P. S. Doyle, and S. Garaj, Nat. Commun. 10, 1 (2019).

[11] W. R. Taylor, Nature (London) 406, 916 (2000)

[12] M. Jamroz, W. Niemyska, E. J. Rawdon, A. Stasiak, K. C. Millett, P. Sułkowski, and J. I. Sulkowska, Nucleic Acids Res. 43, D306 (2014).

[13] N. C. Lim and S. E. Jackson, J. Phys.: Condens. Matter 27, 354101 (2015).

[14] F. Ziegler, N. C. Lim, S. S. Mandal, B. Pelz, W.-P. Ng, M. Schlierf, S. E. Jackson, and M. Rief, Proc. Natl. Acad. Sci. USA 113, 7533 (2016).

[15] A. Bustamante, J. Sotelo-Campos, D. G. Guerra, M. Floor, C. A. Wilson, C. Bustamante, and M. Báez, Nat. Commun. 8, 1581 (2017).

[16] A. Y. Grosberg and Y. Rabin, Phys. Rev. Lett. 99, 217801 (2007).
[17] L. Dai, C. B. Renner, and P. S. Doyle, Macromolecules 47, 6135 (2014).

[18] L. Dai, C. B. Renner, and P. S. Doyle, Macromolecules 48, 2812 (2015).

[19] L. Dai and P. S. Doyle, Macromolecules 49, 7581 (2016).

[20] L. Dai and P. S. Doyle, Macromolecules 51, 6327 (2018).

[21] J. Tang, N. Du, and P. S. Doyle, Proc. Natl. Acad. Sci. USA 108, 16153 (2011).

[22] C. B. Renner and P. S. Doyle, Soft Matter 11, 3105 (2015).

[23] L. Dai, B. W. Soh, and P. S. Doyle, Macromolecules 52, 6792 (2019).

[24] D. Marenduzzo, C. Micheletti, E. Orlandini, and D. W. Sumners, Proc. Natl. Acad. Sci. USA 110, 20081 (2013).

[25] T. Christian, R. Sakaguchi, A. P. Perlinska, G. Lahoud, T. Ito, E. A. Taylor, S. Yokoyama, J. I. Sulkowska, and Y. Hou, Nat. Struct. Mol. Biol. 23, 941 (2016).

[26] Á. San Martín, P. Rodriguez-Aliaga, J. A. Molina, A. Martin, C. Bustamante, and M. Baez, Proc. Natl. Acad. Sci. USA 114, 9864 (2017).

[27] V. Marcos, A. J. Stephens, J. Jaramillo-Garcia, A. L. Nussbaumer, S. L. Woltering, A. Valero, J.-F. Lemonnier, I. J. Vitorica-Yrezabal, and D. A. Leigh, Science 352, 1555 (2016).

[28] R. Everaers, S. K. Sukumaran, G. S. Grest, C. Svaneborg, A. Sivasubramanian, and K. Kremer, Science 303, 823 (2004)

[29] L. Dai, J. J. Jones, J. R. van der Maarel, and P. S. Doyle, Soft Matter 8, 2972 (2012).

[30] See Supplemental Material at http://link.aps.org/supplemental/ 10.1103/PhysRevResearch.2.022014 for more simulation results and analysis.

[31] P. Grassberger, Phys. Rev. E 56, 3682 (1997).

[32] L. Dai, J. van der Maarel, and P. S. Doyle, Macromolecules 47, 2445 (2014). 
[33] L. Dai, C. B. Renner, and P. S. Doyle, Adv. Colloid Interface Sci. 232, 80 (2016).

[34] M. Frank-Kamenetskii and A. Vologodskii, Sov. Phys. Usp. 24, 679 (1981)

[35] O. Vandans, K. Yang, Z. Wu, and L. Dai, Phys. Rev. E 101, 022502 (2020).

[36] L. Tubiana, E. Orlandini, and C. Micheletti, Prog. Theor. Phys. Suppl. 191, 192 (2011).

[37] L. Tubiana, A. Rosa, F. Fragiacomo, and C. Micheletti, Macromolecules 46, 3669 (2013).
[38] L. Coronel, E. Orlandini, and C. Micheletti, Soft Matter 13, 4260 (2017).

[39] P. Pierański, S. Przybył, and A. Stasiak, Eur. Phys. J. E 6, 123 (2001).

[40] T. Odijk, Macromolecules 16, 1340 (1983).

[41] Y. Yang, T. W. Burkhardt, and G. Gompper, Phys. Rev. E 76, 011804 (2007).

[42] P. Poier, C. N. Likos, and R. Matthews, Macromolecules 47, 3394 (2014).

[43] E. Orlandini, M. Baiesi, and F. Zonta, Macromolecules 49, 4656 (2016). 